\title{
Terahertz Diagnostics and Treatment (TDT-2009) Conference at the Second International Symposium on Topical Problems of Biophotonics-2009
}

\author{
A. P. Shkurinov \\ Department of Physics and International Laser Center, Lomonosov Moscow State University, \\ Moscow, 119992 Russia
}

DOI: $10.1134 / \mathrm{S} 0030400 \mathrm{X} 10060019$

The history of optics and optoelectronics in the terahertz frequency range (millimeter and submillimeter wavelengths) began with the pioneering works of H.R. Hertz, J.C. Bose, and A.S. Popov. Initially, this science was developed in the interests of astronomy, astrophysics, and the physics of plasma. Fields of research such as the generation and various applications of terahertz radiation obtained using synchrotron accelerators, free-electron lasers, and lamp sources; studying the propagation of terahertz radiation in free space; terahertz lidars, and developing new principles of information transmission in the terahertz frequency range can already be considered mature. The early 21 st century has been characterized by the creation of new powerful sources of pulsed terahertz radiation and ultrasensitive methods of its detection, which initiated the development of several fundamentally new directions that could not previously be attributed to this frequency range. In these directions, the application of terahertz radiation in biology and medicine, the study of chemical reactions in real time, and the development methods of the formation of informative confocal images and tomography undoubtedly should belong.

The first special issue of Optika i Spectroskopiya (Optics and Spectroscopy), which was devoted to the discussion of modern problems of terahertz optics, optoelectronics, and spectroscopy, was published in 2009 (Opt. Spectrosc. 107 (4)) and included six papers that presented results obtained by scientific groups at Moscow State University; St. Petersburg State University of Information Technologies, Mechanics, and Optics; the Institute of Spectroscopy of the Russian Academy of Sciences; and the Institute of Laser Physics, Siberian Branch of the Russian Academy of Sciences. Previously, the authors of these papers presented lectures on invitation at the 5th International Congress on the Fundamental Problems of Optics
2008: Terahertz Optics and Spectroscopy, which was held in October of 2008 at St. Petersburg State University of Information Technologies, Mechanics, and Optics. The papers published in that issue reported on the progress in this intensively developing area and attracted significant interest from the Russian scientific community.

It gives me great pleasure to present to the readers the second special issue of the journal, which is devoted to the problems of modern terahertz optics, optoelectronics, and spectroscopy. This issue includes five papers, whose authors presented invited lectures at the Conference Terahertz Diagnostics and Treatment (TDT-2009), which was held July 19-24, 2009 at the Second International Symposium Topical Problems of Biophotonics 2009 at the Institute of Applied Physics of the Russian Academy of Sciences in Nizhni Novgorod. Despite their small volume, these papers give fairly full coverage to modern trends in the development of this direction. Based on the reports included in this thematic issue of the journal, one can make the conclusion that, at present, terahertz optics and spectroscopy, as an interdisciplinary avenue of research, has widely developed in this country. Year after year, the scientific community focused on this field substantially increases due to the involvement of young scientists and the organization of new research groups. It becomes evident that researchers and engineers that work in various allied fields of knowledge are actively searching for ways to use electromagnetic radiation in the terahertz frequency range in previously developed optical technologies aimed at obtaining new knowledge and materials. 